\title{
A NEW APPROACH TO MINIMIZE STABILITY FACTOR OF AN
}

\section{AMPLIFIER}

\author{
Puneet Sharma $^{1}$, Atul Goyal ${ }^{2}$, Mahendra Pindel ${ }^{3}$ \\ ${ }^{1,2,3}$ M.TECH SCHOLAR, Dr B. R. Ambedkar National Institute of Technology Jalandhar, Punjab, India
}

\begin{abstract}
In this paper, a method is proposed for minimizing stability factor and removing the negative feedback from Self Bias Configuration of an Amplifier. Stability is an important concern in the designing of Amplifier. For stabilization of an Amplifier different biasing techniques are used. Among all biasing techniques Self Bias technique is best but the major drawback is the introduction of negative feedback by Emitter resistance $R_{e}$. Due to the introduction of negative feedback the gain of an amplifier decreases. An ideal Ammeter has infinite internal resistance so to remove the negative feedback an ideal Ammeter is used instead of Emitter resistance $R_{e}$. With the application of proposed method the Stability factor is minimized and coming out to be equal to Unity and the problem of negative feedback is also removed. In this paper the analysis of proposed Self Bias Circuit is done with the help of Thevenin Theorem.
\end{abstract}

Keywords: Negative Feedback, Stability Factor, Self-Bias Circuit, Amplifier

\section{INTRODUCTION}

Transistors are used as amplifiers, oscillators, switching circuits etc. However, the principal use of transistor is as an amplifier [1]. An amplifier increases the magnitude of a given signal applied to its input. Most of the transistor amplifiers are required to work as linear amplifier. An amplifier is said to be linear if its output voltage is a linear function of its input voltage. Such a linear operation is ensured when zero signal operating point is selected properly, desirably in the middle of the active region and the operation is restricted in the linear region of the characteristic curves, thereby avoiding the distortion of the signal waveform [2]. Thus selection of zero signal operating point is of great importance and may be done by using suitable biasing arrangement i.e. by applying proper dc voltages to emitter-to-base junction and collector-to-base junction. The biasing circuit used in transistor should be such as:

i. To establish conveniently the operating point in the middle of the active region of the characteristics.

ii. To make the operating point independent of transistor parameters

iii. To stabilize the collector current against temperature variations.

\section{MATERIALS AND METHODS}

\subsection{Stability Factor}

Stability Factor $\mathrm{S}$ indicates the variation in collector current $I_{c}$ due to variation in reverse saturation current $I_{c o}$ because of variation in Temperature. Smaller the value of $S$, better will be the thermal stability. In Ideal case the minimum value of $S=1$ and in Practical case, the minimum value of $\mathrm{S}=1.3$. If $\mathrm{S}$ is less than $1+\beta$ then the amplifier is stable otherwise unstable [3

$$
S=\frac{\partial I_{c}}{\partial I_{c o}}
$$

Where $V_{B E}$ and $\beta$ are constant.

\section{General equation of Stability Factor S}

$$
I_{c}=\beta I_{b}+(1+\beta) I_{c o}
$$

Differentiating both side with respect to $I_{c}$

$$
1=\beta \frac{\partial I_{b}}{\partial I_{c}}+(1+\beta) \frac{\partial I_{c o}}{\partial I_{c}}
$$

Using Equation (1) in equation (3), we get

$$
1=\beta \frac{\partial I_{b}}{\partial I_{c}}+(1+\beta) \frac{1}{S}
$$

On simplifying Equation (4), we get

$$
S=\frac{1+\beta}{1-\beta \frac{\partial I_{b}}{\partial I_{c}}}
$$

\subsection{Algorithm to Find Stability Factor S}

Step 1: apply KVL to the input Mesh to obtain input Equation. Step 2: Substitute $I_{e}=I_{c}+I_{b}$ in input equation and simplify.

Step 3: differentiate the equation obtained above with respect to $I_{C}$ keeping $V_{B E}$ and $\beta$ as constant.

Step 4: simplify the obtained equation to get $\frac{\partial I_{b}}{\partial I_{c}}$. 
Step 5: substitute the value of $\frac{\partial I_{b}}{\partial I_{c}}$ in general equation of $\mathrm{S}$.

\subsection{Biasing Circuits}

There are three basic Biasing Circuits

\subsubsection{Fixed Bias Circuit}

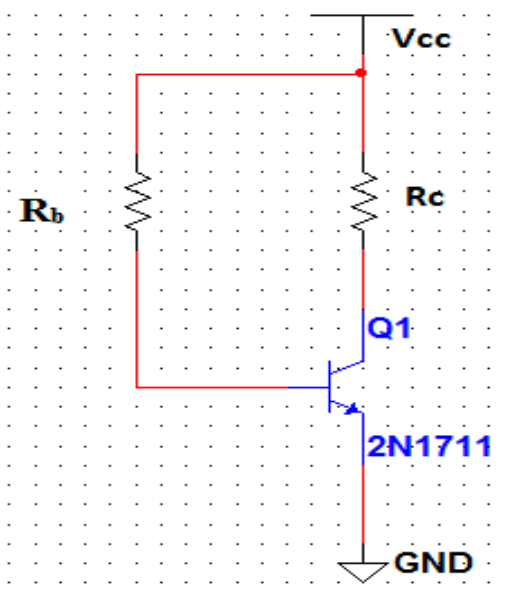

Fig -1: Fixed Bias Circuit

In fixed bias circuit the stability factor

$$
\mathrm{S}=1+\beta
$$

$\mathrm{S}$ is very high that is poor thermal stability.

\subsubsection{Collector to Base Bias Circuit}

In collector to Base bias circuit Stability factor

$$
S=\frac{1+\beta}{1+\beta \frac{R_{c}}{R_{c}+R_{b}}}
$$

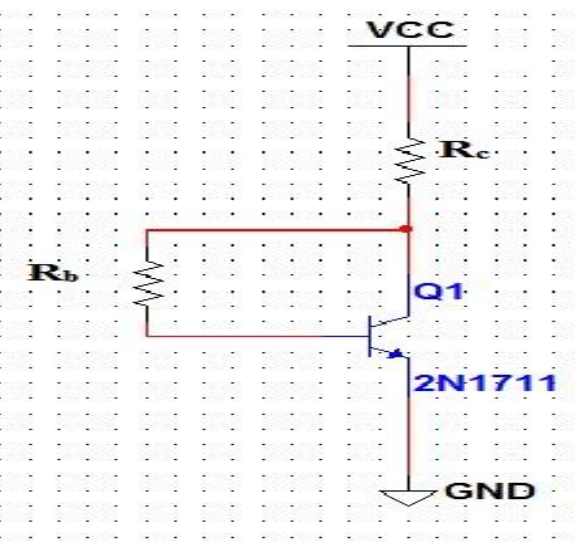

Fig -2: Collector to Base Bias Circuit
$\mathrm{S}$ is small that is the circuit is having good thermal stability. The function of base resistance $R_{b}$ is to provide a base current to the transistor and since it is connected between collector and base it will introduce the unwanted negative feedback and reduces the voltage gain of the amplifier. This biasing circuit is also practically rejected.

\subsubsection{Self Bias Circuit}

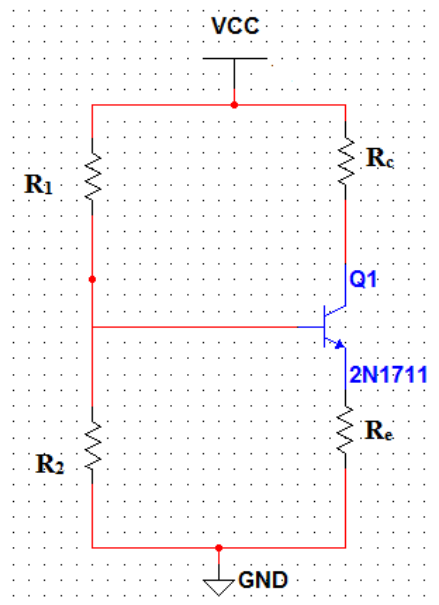

Fig -3: Self Bias Circuit

Self-bias circuit is also called potential divider or emitter bias circuit. This is the popularly used biasing circuit. The BJT is in CE mode with emitter resistance $R_{e}$ and will introduce $180^{\circ}$ phase shift for the input signal. $\mathrm{R}_{1}$ and $\mathrm{R}_{2}$ are biasing resistors and they are used to provide a base current into the transistor ( $R_{1} \geq 10 R_{2}$ ). Simplified Self-bias Circuit using Thevenin Theorem.

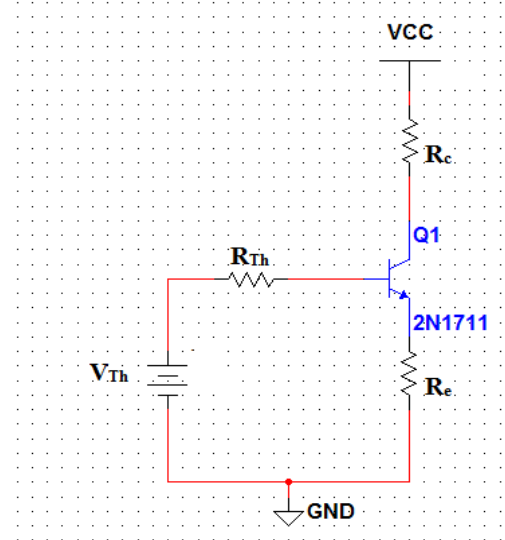

Fig -4: Thevenin Equivalent of Self Bias Circuit

Thevenin open circuit voltage

$$
\mathrm{V}_{\mathrm{Th}}=\mathrm{V}_{\mathrm{cc}}\left(\frac{R_{2}}{R_{1}+R_{2}}\right)
$$


And Thevenin Internal resistance

$$
\mathrm{R}_{\mathrm{Th}}=\mathrm{R}_{1} \| \mathrm{R}_{2}
$$

On applying KVL in input Mesh

$$
\begin{gathered}
V_{T h}=I_{b} R_{T h}+V_{B E}+I_{e} R_{e} \\
\text { Substitute } I_{e}=I_{c}+I_{b}
\end{gathered}
$$

and on simplifying, we get

$$
\mathrm{V}_{\mathrm{Th}}=\mathrm{I}_{\mathrm{b}} \mathrm{R}_{\mathrm{Th}}+\mathrm{V}_{\mathrm{BE}}+\mathrm{I}_{\mathrm{c}} \mathrm{R}_{\mathrm{e}}+\mathrm{I}_{\mathrm{b}} \mathrm{R}_{\mathrm{e}}
$$

On differentiating with respect to $I_{c}$ we get

$$
0=\left(\mathrm{R}_{\mathrm{Th}}+\mathrm{R}_{\mathrm{e}}\right) \frac{\partial \mathrm{I}_{\mathrm{b}}}{\partial \mathrm{I}_{\mathrm{c}}}+\mathrm{R}_{\mathrm{e}}
$$

On simplifying,

$$
\frac{\partial \mathrm{I}_{\mathrm{b}}}{\partial \mathrm{I}_{\mathrm{c}}}=\frac{-\mathrm{R}_{\mathrm{e}}}{\mathrm{R}_{\mathrm{Th}}+\mathrm{R}_{\mathrm{e}}}
$$

On substituting the above equation in equation (1)

$$
S=\frac{1+\beta}{1+\beta \frac{\mathrm{R}_{\mathrm{e}}}{\mathrm{R}_{\mathrm{Th}}+\mathrm{R}_{\mathrm{e}}}}
$$

$\mathrm{S}$ is very small and that is self- bias circuit is having excellent thermal stability.

Self- bias circuit with emitter resistance $R_{e}=0$ is unstable and stability factor becomes

$$
\mathrm{S}=1+\beta
$$

That is $\mathrm{R}_{\mathrm{e}}$ is mainly responsible for providing the thermal stability or Q-point stability in the circuit hence $R_{e}$ is called the Stabilization Resistance. The major disadvantage of using emitter resistance $R_{e}$ is that negative feedback is introduced in the circuit due to which gain decreases.

\subsection{Proposed Biasing Circuit}

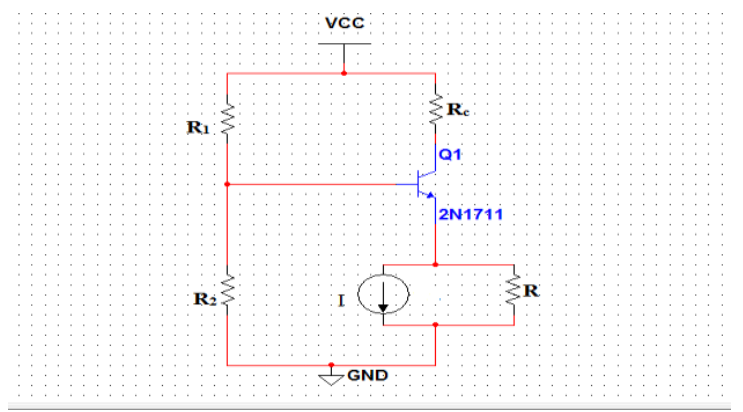

Fig -5: Proposed Biasing Circuit
To eliminate the problem of negative feedback across $R_{e}$, a practical current source is connected in place of emitter resistance so that the internal resistance of current source which is very large will be working as an emitter resistance in the circuit. By this the problem of negative feedback in the circuit is eliminated.

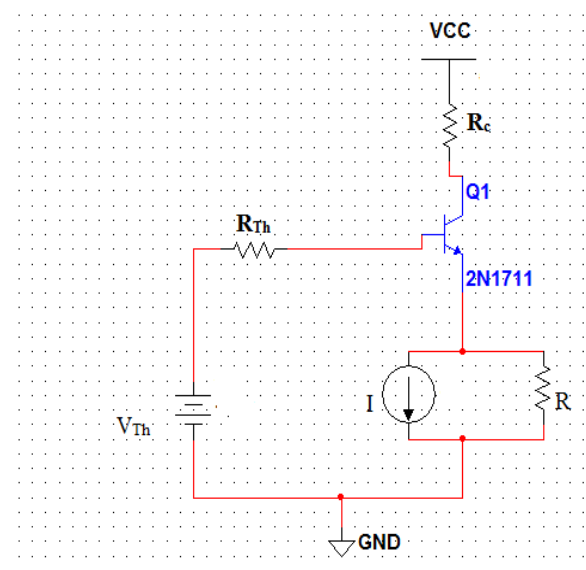

Fig -6: Thevenin Equivalent of Proposed Biasing Circuit

On applying KVL in input Mesh

$$
\begin{gathered}
V_{T h}=I_{b} R_{T h}+V_{B E}+R I_{R} \\
\text { Substitute } I_{R}=I_{e}-I_{A}
\end{gathered}
$$

and on simplifying, we get

$$
\mathrm{V}_{\mathrm{Th}}=\mathrm{I}_{\mathrm{b}} \mathrm{R}_{\mathrm{Th}}+\mathrm{V}_{\mathrm{BE}}+\mathrm{I}_{\mathrm{e}} \mathrm{R}-\mathrm{I}_{\mathrm{A}} \mathrm{R}
$$

On differentiating with respect to $I_{c}$ we get

$$
0=\left(\mathrm{R}_{\mathrm{Th}}+\mathrm{R}\right) \frac{\partial \mathrm{I}_{\mathrm{b}}}{\partial \mathrm{I}_{\mathrm{c}}}+\mathrm{R}
$$

On simplifying,

$$
\frac{\partial \mathrm{I}_{\mathrm{b}}}{\partial \mathrm{I}_{\mathrm{c}}}=\frac{-\mathrm{R}}{\mathrm{R}_{\mathrm{Th}}+\mathrm{R}}
$$

On substituting the above equation in equation (1),

$$
\begin{gathered}
S=\frac{1+\beta}{1+\beta \frac{\mathrm{R}}{\mathrm{R}_{\mathrm{Th}}+\mathrm{R}}} \\
\mathrm{S}=\frac{(1+\beta)\left(\mathrm{R}+R_{T h}\right)}{(1+\beta) \mathrm{R}+R_{T h}}
\end{gathered}
$$

As $\mathrm{R}$ is the internal Resistance of Ideal current source therefore $\mathrm{R}$ is very high $(R \rightarrow \infty)$. 
On applying this condition on Equation (10), we get

$$
\mathrm{S}=\lim _{R \rightarrow \infty} \frac{(1+\beta)\left(\mathrm{R}+R_{T h}\right)}{(1+\beta) \mathrm{R}+R_{T h}}
$$

On solving, we get

$\mathrm{S}=1$.

\section{CONCLUSIONS}

In this paper, we have proposed a method to stabilize the amplifier with the help of an ideal Ammeter so that the problem of negative feedback is also removed. An interesting by-product of this work is that the minimum stability factor i.e. $S=1$ is achieved.

\section{REFERENCES}

[1]. Ghandhi, S.K. "Bias Considerations in Transistor Circuit Design"Circuit Theory, IRE Transactions on (Volume:4, Issue: 3 )Sep 1957 Page-194 - 202.

[2]. Gaur, S.P. ,Navon, D.H. , Teerlinck, R.W. "Transistor design and thermal stability"Published in: Electron Devices, IEEE Transactions on (Volume:20 , Issue: 6 )Jun 1973 Page:527 - 534 .

[3]. Sarmiento-Reyes, A. "A novel method to predict both the upper bound on the number and the stability of DC operating points of transistor circuits"Circuits and Systems, 1995. ISCAS '95., 1995 IEEE International Symposium on (Volume:1 ) 1995 Page:101 - 104 vol1.

\section{BIOGRAPHIES}

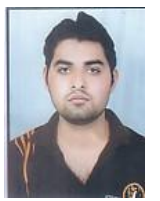

Puneet Sharma has completed his B. Tech in Electronics and Communication Engineering from JNIT, Jaipur in 2010 and is currently pursuing his M. Tech in Dr. B. R. Ambedkar National Institute of Technology, Jalandhar. His area of interest includes Signal Processing, Electronic Devices, Communication System and Control Engineering.

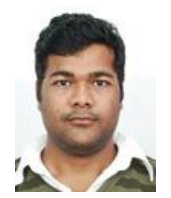

AtulGoyal has received his B. Tech degree in Electronics and Communication Engineering from VIT University in 2011 and is currently pursuing his M. Tech in Dr. B. R. Ambedkar National Institute of Technology, Jalandhar.

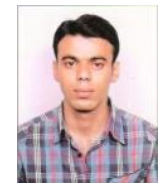

MahendraPindel has completed his B. Tech in Electronics and Communication Engineering from Marudhar Engineering College, Bikaner in 2011 and is currently pursuing his M. Tech in Dr. B. R. Jalandhar. Ambedkar National Institute of Technology, 